\title{
Parásitos gastrointestinales de hicoteas Trachemys callirostris (Emydidae) traficadas ilegalmente en Córdoba, Colombia
}

\author{
Gastrointestinal parasites in Colombian sliders Trachemys callirostris (Emydidae) \\ illegally trafficked in Córdoba, Colombia \\ Rafael David Blanco Martínez ${ }^{1}$, Hernán Padilla Cordero ${ }^{1}$, Juan Carrascal \\ Velásquez $^{2}$, César Rojano Bolaño ${ }^{3}$, Gabriel Álvarez Otero ${ }^{4}$, Santiago Monsalve ${ }^{5}$, \\ Alberto Mestra ${ }^{6}$, Julio Chacón-Pacheco ${ }^{7,8}$
}

\section{Resumen}

\begin{abstract}
Se determinó la presencia de parásitos gastrointestinales en el tracto gastrointestinal de 100 hicoteas de la especie Trachemys callirostris ingresadas al Centro de Atención y Valoración de Fauna Silvestre (CAV), Córdoba, Colombia, durante los años 2009 y 2010 , como producto de decomisos del tráfico ilegal de animales silvestres. Los tractos gastrointestinales fueron separados, seccionados y lavados y el contenido fie removido para identificar y cuantificar los parásitos. Se aislaron 1064 individuos de la especie Nematophila argentinum (44\%) y de los géneros Serpinema (44\%) y Spiroxys (12\%).
\end{abstract}

1 Grupo de Estudio de Fauna Silvestre GEDFAS, Facultad de Medicina Veterinaria y Zootecnia, Universidad de Córdoba, Montería, Córdoba, Colombia

2 OHLAIC/One Health Colombia. Grupo GIPAT/ Grupo de estudio de Fauna Silvestre GEDFAS y Bienestar Animal YGIIS, Departamento de Ciencias Pecuarias, Facultad de Medicina Veterinaria y Zootecnia, Universidad de Córdoba, Montería, Córdoba, Colombia

3 Fundación Cunaguaro, Yopal, Casanare, Colombia

4 In Situ Consultores SAS, Magdalena, Colombia

5 Corporación Universitaria Lasallista, Facultad de Ciencias Administrativas y Agropecuarias, Caldas, Antioquia, Colombia

${ }^{6}$ Programa de Bacteriología, Facultad de Ciencias de la Salud, Universidad de Córdoba, Montería, Córdoba, Colombia

${ }^{7}$ Grupo de Investigación Biodiversidad Unicórdoba, Universidad de Córdoba, Montería, Córdoba, Colombia

8 E-mail: jchacon_bio@hotmail.com

Fuente de financiación: El estudio es parte del Proyecto «Formulación e implementación de un plan de manejo en poblaciones de hicoteas in situ y ex situ, para promover la conservación de la especie en el departamento de Córdoba - Colombia» financiado por el Centro de Investigaciones de la Universidad de Córdoba

Recibido: 22 de agosto de 2020

Aceptado para publicación: 15 de enero de 2021

Publicado: 24 de abril de 2021 
Las prevalencias encontradas fueron de 70\% para $N$. argentinum (Trematoda) y de 63 y $39 \%$ para Serpinema sp y Spiroxys sp (Nematoda), respectivamente. La intensidad media fue de 6.7 para $N$. argentinum, 7.3 para Serpinema sp y de 3.2 para Spiroxys sp. $N$. argentinum se encontró mayormente en intestino grueso (40\%); sin embargo, este es el primer reporte de este parásito en boca, esófago y cloaca. Los individuos del género Serpinema sp, se encontraron inmersos en el contenido del tracto digestivo, con mayor proporción en intestino delgado (63\%), mientras que Spiroxys sp se localizaron principalmente en estómago (64\%). Las lesiones encontradas fueron localizadas y probablemente no comprometían significativamente la vida de los individuos.

Palabras clave: helmintos, Nematophila argentinum, Serpinema, Spiroxys, tortuga de agua dulce

\section{AbSTRaCt}

The presence of gastrointestinal parasites in gastrointestinal tract of 100 Colombian sliders (Trachemys callirostris) admitted to the Wildlife Care and Assessment Center (CAV), Córdoba, Colombia, during 2009 and 2010, as a result of confiscations from illegal trafficking of wild animals. The gastrointestinal tracts were separated, washed and its contents were removed to identify and quantify the parasites. A total of 1064 individuals of the taxa Nematophila argentinum (44\%), Serpinema sp (44\%) and Spiroxys sp (12\%) were isolated. The observed prevalences were $70 \%$ for $N$. argentinum (Trematode), and 63 and 49\% for Serpinema sp and Spiroxys sp (Nematode), respectively. The mean intensity was 6.7 for N. argentinum, 7.3 for Serpinema sp, and 3.2 for Spiroxys sp. N. argentinum was mainly found in the large intestine (40\%); however, this is the first report of this parasite in the mouth, oesophagus and cloaca. Individuals of Serpinema sp were found mixed within the content of the digestive tract, especially in the small intestine (63\%), whereas Spiroxys sp were located mainly in the stomach $(64 \%)$. The lesions found were focused and without compromising the lives of turtles.

Key words: helminths, Nematophila argentinum, Serpinema, Spiroxys, freshwater turtles

\section{INTRODUCCIÓN}

Las tortugas del género Trachemys presentan una taxonomía dinámica, donde se han reconocido cambios sustanciales en los últimos 10 años (TTWG et al., 2017). En el norte de América del Sur se reconocen las especies T. medemi y T. venusta, esta última con dos subespecies: T. v. callirostris en el norte de Colombia y noreste de Venezuela, y T. v. chichiriviche al oeste de la costa venezolana (TTWG et al., 2017, Vargas Ramírez et al., 2017). Sin embargo, debido a la falta de análisis que involucren datos moleculares adicionales y un muestreo geográfico más profundo de especímenes verificados en el campo, para el propósito de este trabajo se utilizará el nombre de Trachemys callirostris en referencia a la hicotea del norte de Colombia (TTWG et al., 2017).

La hicotea es una tortuga de agua dulce, de tamaño moderado (longitud del caparazón $=33 \mathrm{~cm}$; Bock et al., 2010). Es uno de los quelonios más explotados en Colombia, dado que la carne, el hígado, los huevos y los folículos ováricos son altamente apreciados por su buen sabor (Castaño-Mora, 2002); además, los neonatos son vendidos como 
mascotas en las principales ciudades de Colombia (Castaño-Mora, 2002). Esta sobreexplotación ha ocasionado una merma importante en la población de esta especie (De la Ossa y Riaño, 1999; Palacios et al., 1999), razón por la cual se ha categorizado como una especie vulnerable a la extinción en el país (Ministerio de Ambiente y Desarrollo Sostenible, 2017), haciendo que se tomen medidas de control policivo, reflejadas en los grandes volúmenes de hicoteas que llegan a los centros de atención de fauna silvestre, procedentes del decomiso producto de la comercialización ilegal de estos animales (De la Ossa, 2003; Mendivelso y Montenegro, 2007; CVS, 2009).

A pesar de los grandes volúmenes de decomisos de hicoteas, son pocos los trabajos que se han desarrollado en el país, orientados a dar pautas sobre el manejo biológico y médico de esta especie en cautiverio (De la Ossa y Riaño, 1999; Barragán, 2002; Bonilla et al., 2012; Carrascal et al., 2014). Las condiciones de cautiverio generan estrés, concentración de un gran número de individuos y presencia de varias especies en un mismo encierro; pudiendo convertirse en catalizadores del desarrollo, multiplicación y propagación de parásitos y otros microorganismos (García, 2013), por causa de una depresión de la respuesta inmune (Rataj et al., 2011).

Los endoparásitos pueden generar pérdida de peso y condición corporal, anemia, enteritis, y lesiones inflamatorias y ulcerativas, entre otras (Yarto, 2011; Martínez-Silvestre et al., 2013). No obstante, la carencia de buenos métodos de diagnóstico, la escasez de laboratorios clínicos en los cuales se realicen las técnicas correctas, y la falta de conocimiento acerca de la fauna parasítica normal de la mayoría de los taxones de reptiles, incluidas las tortugas, dificulta el diagnóstico de enfermedades en estas especies (Pasmans et al., 2008). Por esta razón, se requiere de estudios que permitan conocer las principales parasitosis en hicoteas (T. callirostris), tanto en vida silvestre como en cautiverio.
Estudios en Colombia han determinado la presencia de helmintos en esta especie en vida silvestre, entre ellos las especies Heronimus mollis, Telorchis corti y Nematophila argentinum en el Río Magdalena (Lenis y Vélez, 2011), Nematophila argentinum y Ancyracanthus sp en la cuenca del río Sinú, en Córdoba (Mestra y Requena, 2004) y Polystomoides magdalenensis en el Río Magdalena (Lenis y García-Prieto, 2009). En condiciones en cautiverio, se reportan los parásitos Balantidium sp, Entamoeba invadens, Tricomonas sp, Eimeria sp, e Isospora sp (Barragán, 2002), haciéndose evidente la falta de información al respecto.

Teniendo en cuenta lo anterior, el objetivo de este trabajo fue identificar los parásitos gastrointestinales de hicoteas adultas ( $T$. callirostris) que ingresaron al Centro de Atención y Valoración de Fauna Silvestre (CAV-CVS) de Montería, Córdoba, producto del tráfico ilegal de fauna silvestre.

\section{Materiales y Métodos}

Las muestras correspondieron a tractos gastrointestinales obtenidos de la necropsia de hicoteas adultas que murieron en cautiverio en el Centro de Atención y Valoración de Fauna Silvestre (CAV), de la Corporación Autónoma Regional de los Valles del Sinú y San Jorge (CVS), ubicado en Montería, Córdoba, Colombia, durante los años 2009 y 2010. Este centro se encuentra ubicado en la ciudad de Montería, departamento de Córdoba (9 $\left.14^{\prime} \mathrm{N} 75^{\circ} 49^{\prime} \mathrm{W}\right)$, a 49 msnm. El tamaño de la muestra fue determinado utilizando un muestreo aleatorio simple, con error del 7.0\% y una confiabilidad del $85 \%$, obteniendo un valor ajustado de 100 animales.

Todos los individuos fueron valorados en el menor tiempo post mortem posible, siguiendo los criterios propuestos por Flint et al. (2009) para quelonios. Para conocer el estado de las tortugas, se calculó la condición corporal mediante el Índice de Fulton (ICC = 
[masa corporal $(\mathrm{kg}) / \mathrm{LE}^{3}(\mathrm{~cm})$ ], donde LE es la longitud (Bjorndal et al., 2000). Solo fue revisado el tracto gastrointestinal en busca de lesiones y parásitos (desde la boca hasta el intestino grueso). Las muestras se obtuvieron manteniendo las normas de bioseguridad establecidas para tal fin. Se tuvo en cuenta la Resolución N. ${ }^{\circ} 008430$ de 1993 (4 de octubre de 1993, Art. 87, literales c, g y h) del Ministerio de Salud de Colombia, con el fin de cumplir con los estándares de bienestar animal y manejo de individuos dentro de investigaciones.

Los tractos gastrointestinales se aislaron por secciones con doble nudo para evitar la migración de parásitos. Los contenidos se colocaron en bolsas de polietileno y conservadas en cavas térmicas con refrigerante (8$12{ }^{\circ} \mathrm{C}$ ) para su transporte. En el Laboratorio de Parasitología de la Facultad de Medicina Veterinaria y Zootecnia de la Universidad de Córdoba se lavó la mucosa de cada una de las porciones con el fin de arrastrar los parásitos presentes. El producto de cada lavado se depositó en frascos rotulados de boca ancha con capacidad de $50 \mathrm{ml}$, agregándole formalina al $10 \%$ en cantidad igual al volumen del lavado realizado, y se conservaron a temperatura de laboratorio para su posterior aislamiento (Vélez, 1999).

Las muestras fueron colocadas en pequeñas cantidades en cajas de Petri y observadas al estereoscopio hasta agotar la totalidad del material. Los parásitos encontrados se depositaron en frascos de boca ancha con capacidad de $20 \mathrm{ml}$ y en solución de glicerina formulada al $5 \%$ para su posterior montaje y clasificación (Vélez, 1999). Para la identificación de los parásitos se utilizaron las claves taxonómicas propuestas por Jones (2005), y Lenis y Vélez (2011) para trematodos, y por Anderson et al. (2009) y Vicente et al (1993) para nematodos. Se determinó la prevalencia, expresada como el número de huéspedes positivos a cada parásito dividido entre el número de huéspedes examinados; y la intensidad media, expresada como el número total de parásitos de cada especie encontrados en el total de la muestra dividido entre el número de huéspedes infectados. Los cálculos fueron basados en los términos ecológicos propuestos por Bush et al. (1997).

\section{Resultados}

De los 100 tractos gastrointestinales se aislaron 1064 helmintos, con una prevalencia del 100\%. De estos, 592 (56\%) correspondieron a nematodos y 472 (44\%) a trematodos. A la evaluación clínica post mortem, el $95 \%$ de las hicoteas se encontraban con alto grado de deshidratación $(>8 \%)$ y baja condición corporal ICC $<1$. Las mucosas donde se encontraban adheridos los parásitos se encontraban congestionadas (Figuras 1A-B). No se encontraron otras lesiones macroscópicas aparentes.

Los trematodos encontrados coincidieron con los individuos de la subfamilia Nematophilinae (Skrjabin, 1949), presentando un cuerpo grande, oval, ancho máximo a nivel del testículo, cutícula lisa. Asimismo, la ventosa oral terminal de 0.5 a $2.5 \mathrm{~mm}$ x 0.5 a $1.5 \mathrm{~mm}$, faringe cilíndrica y muscular con 0.5 a $3.5 \mathrm{~mm}$ de longitud y 0.5 a $1.5 \mathrm{~mm}$ de ancho, esófago corto, asas intestinales bifurcadas, sacos faríngeos extramurales, testículos lobulados y oblicuos, con testículo anterior preecuatorial y el posterior posecuatorial, y el útero intercecal y entre los testículos (Figura 2A), haciéndolo compatible con Nematophila argentinum (Lenis y Vélez, 2011).

Se determinó la presencia de $N$. argentinum en boca (2\%), esófago (4\%), estómago (19\%), intestino delgado $(30 \%)$, intestino grueso $(40 \%)$ y cloaca $(5 \%)$, con una prevalencia de $70 \%$. Los parásitos se encontraron adheridos a la mucosa gastrointestinal (Figura 2B). En los 70 animales infectados con trematodos se aislaron 472 parásitos, para una intensidad media de 6.74 trematodos por animal. 

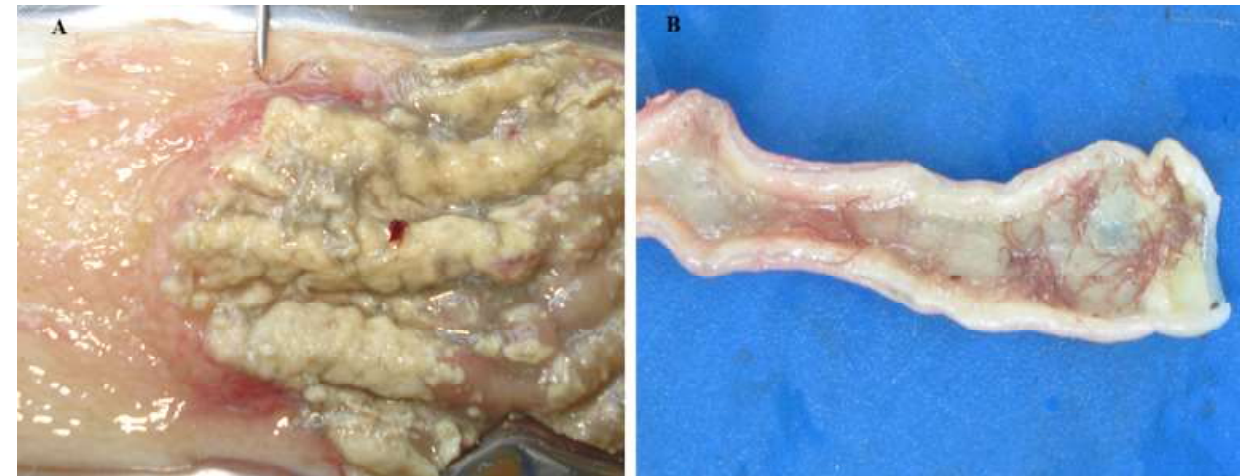

Figura 1.A-B. Lesiones en mucosa gastrointestinal de hicoteas, causadas por nódulos de Spiroxys sp
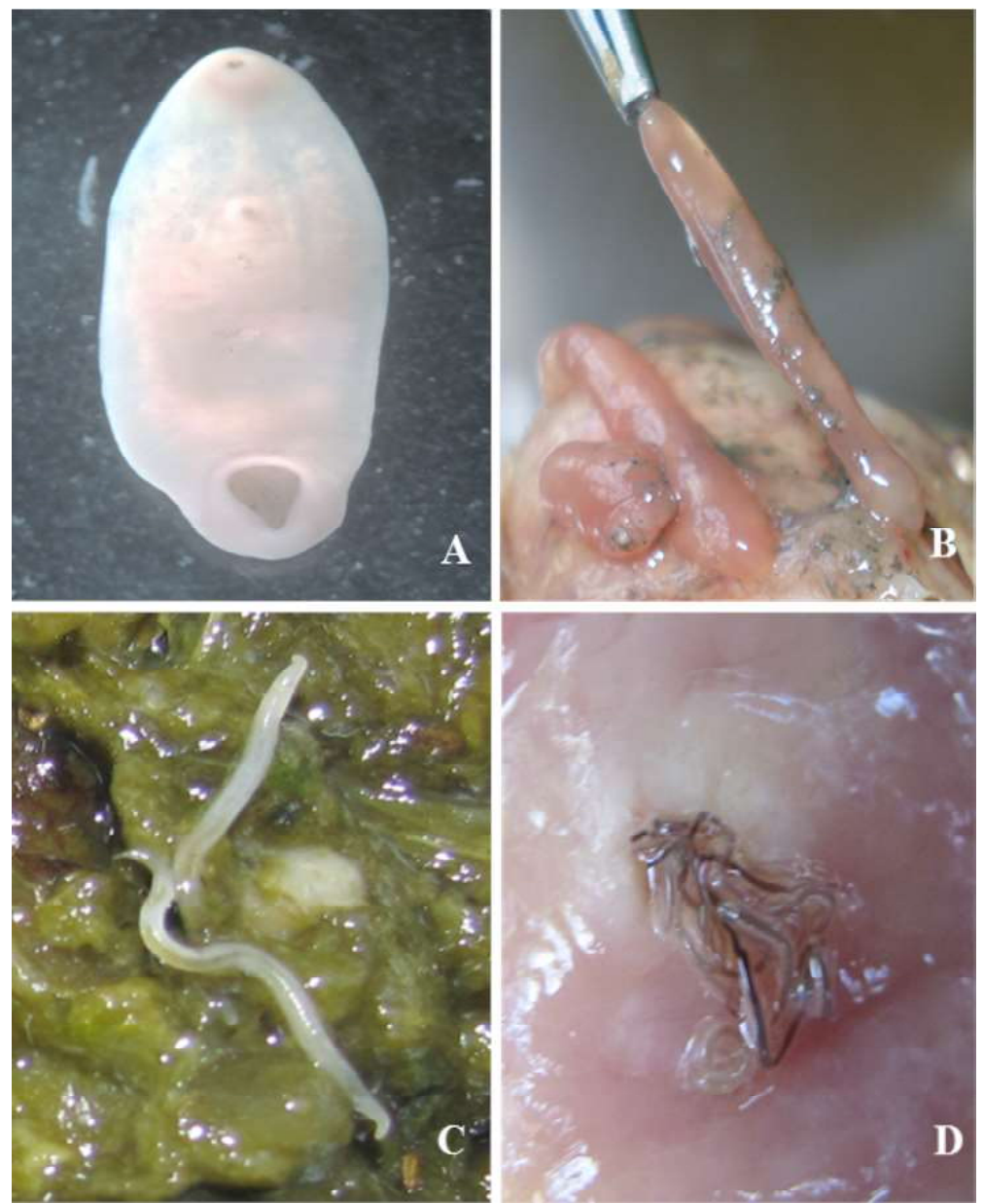

Figura 2. Parásitos gastrointestinales de hicotaeas (Trachemys cacllirostris). A. Nematophila argentinum. B. N. argentinum adherida en intestino grueso. C. Serpinema sp en contenido estomacal. D. Nódulos de Spiroxys sp fijados en mucosa estomacal 

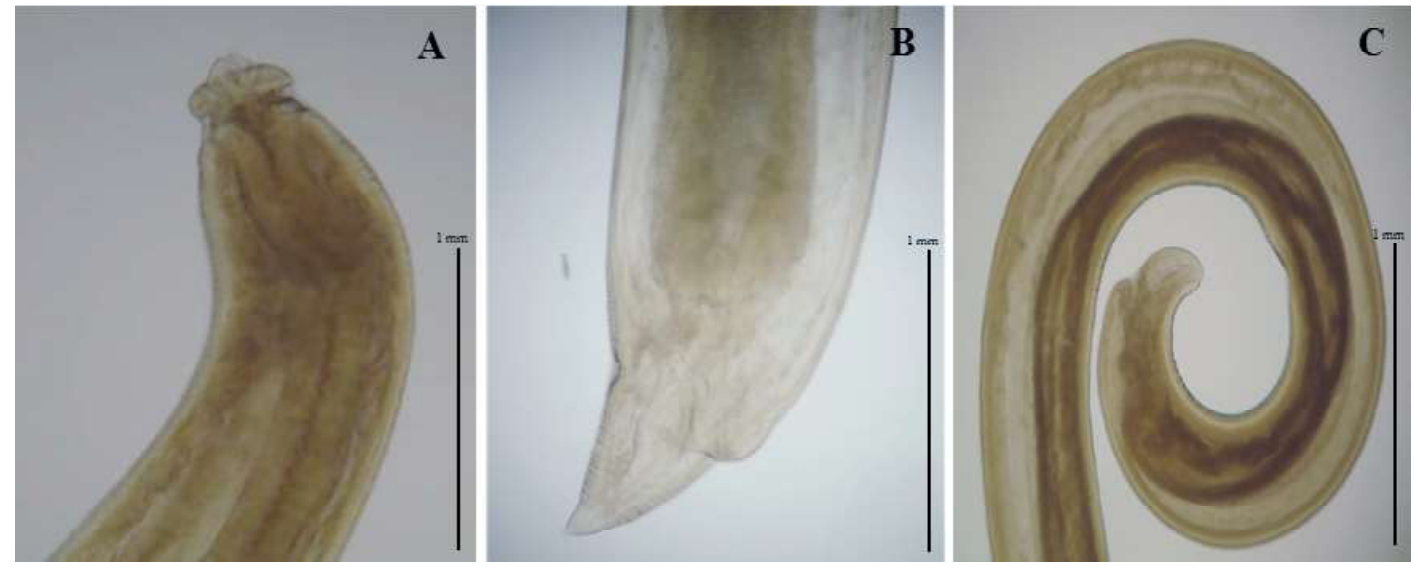

Figura 3. Serpinema sp. A. Cabeza (extremo anterior). B. Cola (extremo posterior) de hembra

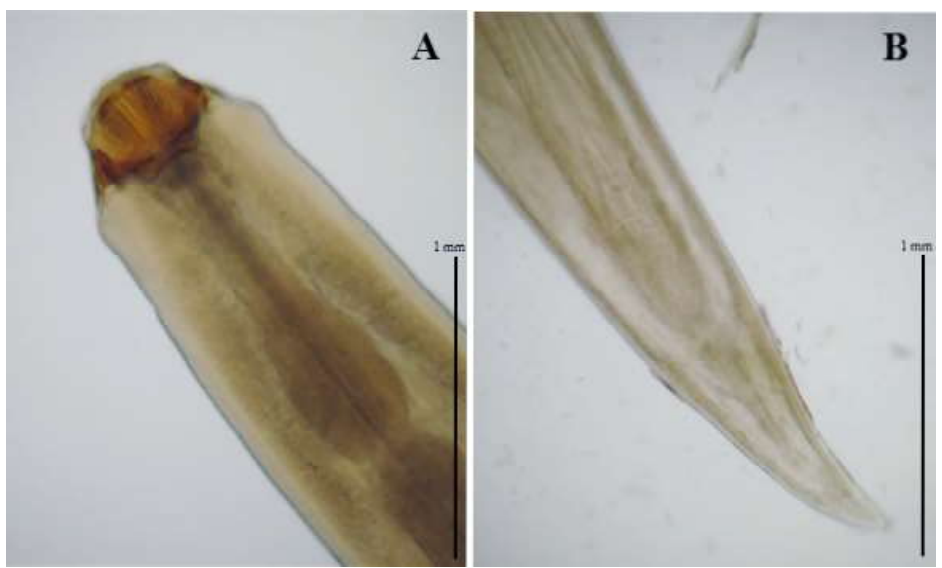

Figura 4. Spiroxys sp. A. Extremo anterior. B. Extremo posterior hembra. C. Extremo posterior macho

De los 592 (56\%) nematodos aislados, el 44\% (466) correspondieron al género Serpinema (Yeh, 1960), quienes morfológicamente se caracterizan como nematodos filiformes. Presentan extremo anterior cortado en ambos sexos. Extremo cefálico provisto de boca bivalva y de dos tridentes. Hembras vivíparas, didelfas, vagina dirigida hacia atrás. Machos con una sola espícula. Gubernáculo ausente. Aletas caudales presentes bien desarrolladas. Cola curvada ventralmente y con fuerte musculatura transversal (Figuras 3A-B).
Los individuos del género Serpinema se encontraron inmersos en el contenido del tracto digestivo, siendo necesario la separación y aislamiento de manera individual del resto de alimentos y heces (Figura 2C). Los parásitos fueron localizados en estómago (14.6\%), intestino delgado (63\%) e intestino grueso $(22.3 \%)$. La prevalencia encontrada fue de $63 \%$. Las 63 hicoteas infectadas presentaban 466 parásitos, con una intensidad media de 7.39 parásitos por animal. 
Por otro lado, el 12\% (126) de los nematodos aislados corresponden al género Spiroxys (Schneider, 1866), siendo nematodos de cuerpo liso, labios grandes, con cutícula interna gruesa y un diente bien definido. Collar cuticular rudimentario o ausente. La cavidad bucal se abre en un corto vestíbulo y presentan esófago cilíndrico. Machos con cola cónica y asa caudal bien desarrollada. Once pares de papilas caudales, dos de los cuales son sésiles y ventrales, anteriores y posteriores a la cloaca. Espículas similares y delicadas. Gubernáculo ausente. Hembras con la vulva en la parte media del cuerpo y vagina dirigida hacia delante. Úteros opuestos (Figuras 3A-B-C).

Los parásitos se localizaron en estómago $(64 \%)$, intestino delgado (1\%) e intestino grueso $(35 \%)$. Se encontraron adheridos a la mucosa, creando nódulos donde se fijan muchos individuos causando congestión de la mucosa digestiva (Figura 3B). La prevalencia encontrada fue de $39 \%$, observándose la presencia de 126 helmintos para una intensidad media de 3.23 .

\section{Discusión}

Se reportan dos grupos de parásitos en hicoteas provenientes de decomisos policiales a traficantes ilegales en el departamento de Córdoba: trematodos de la especie $N$. argentinum y nematodos Serpinema sp y Spiroxys sp.

Los endoparásitos son comunes en quelonios que llevan largo tiempo en cautiverio, aún después de tratamientos antiparasitarios, e igualmente comunes en animales de vida libre (García, 2013). No obstante, la incidencia del parasitismo puede variar entre las especies. Martin (1972), reporta que, en general, las tortugas carnívoras acuáticas son más comúnmente parasitadas y llevan una amplia variedad de helmintos del tipo Metazoa
- nematodos, trematodos, acantocéfalos y anélidos, y que los quelonios terrestres son típicamente afectados solo por nematodos, aun cuando algunos trabajos reportan monogeneos, trematodos y garrapatas (Mestra y Requena, 2004; Guglielmone y Nava, 2006; Martínez-Silvestre y Soler, 2008; Lenis y García-Prieto, 2009; Lenis y Vélez, 2011).

Este estudio, al igual que otros realizados en tortugas del género Trachemys, reportan parásitos del género Nematophila (Mestra y Requena 2004; Lenis y Vélez 2011), considerados comunes en tortugas continentales, siendo reportado en México, Perú y Brasil, incluso en varios hospederos (Alho, 1964; Salízar y Sánchez 2004; Lenis y Vélez, 2011). La presencia de este parásito en hicoteas en cautiverio advierte una problemática de manejo de los individuos traficados ilegalmente, debido a que es una especie que puede tener una significativa acción patógena, por la capacidad de alimentarse, como ocurre con otros trematodos, de sangre y otros líquidos tisulares de sus hospedadores (Schmidt y Roberts, 2000). En este estudio se encontraron pequeñas lesiones congestivas en los sitios de adherencia a la mucosa gastrointestinal de $N$. argentinum.

Entre las lesiones más comunes causadas por trematodos que parasitan tortugas se encuentran lesiones cardiovasculares, como aneurismas y arteritis de los grandes vasos, con engrosamientos de las paredes, trombos y hemorragias en la íntima de los vasos, endocarditis mural y valvular y reacción inflamatoria de tipo granulomatoso. Asimismo, dermatitis traumáticas purulentas y ulcerativas, esofagitis, hemorragia petequial en estómago y gastritis ulcerativa (Wilkinson, 2004; Julca et al., 2014). En cuanto a la ubicación de Nematophila, este es el primer reporte de este parásito en boca, esófago y cloaca, siendo reportada anteriormente en estómago e intestinos (Mestra y Requena, 2004; Lenis y Vélez, 2011). 
Los parásitos de los géneros Serpinema y Spiroxys han sido reportados anteriormente en tortugas del género Trachemys (Moravec y Vargas-Vázquez, 1998; Asakawa et al., 2001; Hidalgo et al., 2009; Oi et al., 2012; Martínez-Silvestre et al., 2013); sin embargo, estos reportes son los primeros para la especie T. callirostris y para Colombia. El principal hallazgo macroscópico de lesiones causadas por estos parásitos fue congestión en la mucosa gastrointestinal del huésped (Figura 1B). Se conoce que estas especies de parásitos causan lesiones entéricas inflamatorias y ulcerativas en estómago e intestino (Mcallister et al., 1993; Martínez-Silvestre et al., 2013), y que incluso pueden llegar a causar pancreatitis (Hidalgo-Vila et al., 2011), lo cual afectaría seriamente la salud de tortugas que se encuentren en condiciones inadecuadas de transporte y mantenimiento, como suele ocurrir en centros de valoración de animales decomisados. No obstante, en este estudio no se evidenciaron lesiones macroscópicas mayores, pero tampoco se realizaron pruebas histopatológicas para determinar daños microscópicos.

En cuanto a la localización en el tracto digestivo, Serpinema sp y Spiroxys sp presentan la misma distribución que otros estudios, encontrándose anclados a la mucosa y al contenido digestivo del estómago, intestino delgado e intestino grueso (Moravec y Vargas-Vázquez, 1998; Asakawa et al., 2001; Hidalgo-Vila et al., 2009; Oi et al., 2012; Bernandon et al., 2013; Martínez-Silvestre et al., 2013), causando inflamaciones en todos los animales parasitados. De igual forma, se conoce que las tortugas continentales son los hospederos definitivos de nematodos del genero Spiroxys (Moravec et al., 1995).

El promedio de parásitos por animal fue de 10.64, resultado similar al reportado por Mestra y Requena (2004), quienes registraron 10.43 parásitos por tortuga. La prevalencia de todos los endoparásitos para este estudio fue del $100 \%$, superior a la reportada para otros autores en diversas especies de reptiles (Rataj et al., 2011; García, 2013). La prevalencia encontrada para cada uno de los parásitos se presenta por encima de valores reportados en otros estudios (Platt, 1977; Hidalgo-Vila et al., 2009; Oi et al., 2012; Lenis y Vélez, 2011; Bernardon et al., 2013; Martínez-Silvestre et al., 2013), lo que podría indicar que el transporte y el cautiverio en malas condiciones estarían influyendo en la salud de las hicoteas traficadas en el departamento de Córdoba.

En Colombia se ha reportado el trematodo $N$. argentinum con una prevalencia de $56 \%$ en hicoteas capturadas para la cuenca del Río Magdalena (Lenis y Vélez, 2011), mientras que este trabajo reporta una prevalencia del $70 \%$. Los nematodos del género Spiroxys presentan prevalencias entre el 23 y $29 \%$ en diversas especies de tortugas continentales (Platt 1977; Bernardon et al., 2013), a diferencia del presente trabajo (39\%). Para el caso del género Serpinema, este trabajo reporta una prevalencia de $63 \%$, similar a otros estudios en tortugas Trachemys introducidas en España y Japón que reportan prevalencias entre 45 y $94 \%$ (Hidalgo-Vila et al., 2009, 2011; Oi et al., 2012; MartínezSilvestre et al., 2013).

Aunque las parasitosis se encuentran en equilibrio simbiótico con el hospedador en reptiles de vida libre, este equilibrio se rompe en individuos mantenidos en cautiverio, pudiendo causar enfermedades parasitarias de repercusión clínica. Los endoparásitos pueden ocasionar cuadros de anemia, aunque la consecuencia más importante es la pérdida de peso y condición corporal, así como la asociación con otras etiologías causantes de enteritis (Yarto, 2011).

Debido al gran número de parásitos aislados de Serpinema sp, es probable que los nódulos que forman tengan una acción patológica de importancia sobre las poblaciones de hicotea que llegan al CAV-CVS. La intensidad media de Serpinema sp en $T$. callirostris fue superior a la reportada en 
estado silvestre para Trachemys scripta elegans en Japón, con 2.3 parásitos por individuo (Oi et al., 2012) y en Kinosternon leucostomum en Costa Rica (Bursey y Brooks, 2011), pero inferior a la reportada en España con 17.8 (Hidalgo-Vila et al., 2009). En cuanto al género Spiroxys, la intensidad media fue superior en este estudio a la reportada en K. leucostomum (1) en Costa Rica, pero inferior a la encontrada en $K$. scorpioides (43) en el mismo país (Bursey y Brooks, 2011) y en Phrynops hilarii (12) en Brasil (Bernardon et al., 2013). La intensidad media de $N$. argentinum en hicoteas en este trabajo fue de 6.74, superior a la reportada para esta misma especie en el Río Magdalena (Lenis y Vélez, 2011).

Diversos factores pueden incidir sobre la prevalencia, abundancia e intensidad de las parasitosis. Un aumento en la prevalencia e intensidad media puede estar relacionado con las estrategias de transmisión (Lenis y Vélez, 2011). Se ha descrito que el herbivorismo puede favorecer la infección con parásitos, principalmente del género Serpinema, por ingestión accidental de huéspedes intermedios del parásito que habita en la vegetación acuática, como copépodos y otros crustáceos $\mathrm{y}$, a su vez, la abundancia de estos en el hábitat de la tortuga (Hidalgo-Vila et al., 2009). El cautiverio es otro de los factores a considerar para una mayor intensidad media de parasitosis en reptiles, ya que este puede romper el equilibrio simbiótico entre el parásito y el hospedero, principalmente por las condiciones inadecuadas de manejo, transporte y déficit nutricionales, las cuales pueden llegar a comprometer el sistema inmune de los individuos (García, 2013), similares a las encontradas en los individuos que llegan al CAV-CVS.

\section{Conclusiones}

- Se encontraron parásitos de la especie Nematophila argentinum (44\%) y de los géneros Serpinema (44\%) y Spiroxys (12\%) en el tracto gastroin- testinal de hicoteas (Trachemys callirostris) procedentes de decomisos de tráfico ilegal.

- La prevalencia de parásitos gastrointestinales en $T$. callirostris fue de $70 \%$.

- Las lesiones macroscópicas fueron focalizadas y probablemente no comprometían la vida de los individuos.

\section{Literatura Citada}

1. Alho C. 1964. Dois novos hospedadores de Nematophila grande (Diesing, 1839) (Trematoda: Paramphistomoidea). Bol do Mus Par Emilio Goeldi 52: 1-4.

2. Anderson RC, Chabaud AG, Willmott S. 2009. Keys to the nematode parasites of vertebrates. Wallingford, UK: CABI Publishing. p 334-336.

3. Asakawa M, Suzuki Y, Kimoto Y, Fox MT. 2001. Parasitic nematodes of pet tortoises in Japan: clinical and ecological viewpoints. Proc Assoc Rept Amp Vet 8: 139-144.

4. Barragán K. 2002. Enfermedades de reptiles y anfibios. Bol GEAS 3(1): 18-27.

5. Bernardon F, Valente AL, Müller G. 2013. Gastrointestinal helminths of the Argentine side-necked turtle, Phrynops hilarii (Duméril \& Bibron, 1835) (Testudines, Chelidae), in south Brazil. Pan Am J Aq Sci 8: 55-57.

6. Bock BC, Paez VP, Daza J. 2010. Trachemys callirostris (Gray, 1856) Colombian slider, Jicotea, Hicotea, Galapago, Morrocoy de agua. Chel Res Mon 5: 1-9. doi: 10.3854/crm.5.042.callirostris.v1.2010

7. Bjorndal KA, Bolten AB, Chaloupka $M Y$. 2000. Green turtle somatic growth model: evidence for density dependence. Ecol Applications 10: 269-282. doi: 10.1890/1051-0761(2000)010[0269:GTSGME]2.0.CO;2

8. Bonilla MA, Luque N, Cuervo MA, Barreto LC, Zuluaga C, Vásquez EA. 2012. Tortugas terrestres y de agua dulce de Colombia y manejo de los decomi- 
sos. Bogotá, Colombia: Ministerio de Ambiente y Desarrollo Sostenible. 100 p. [Internet]. Disponible en: http:// www.acherpetologia.org/images/doc/ Cartilla_tortus_manejo_decomisos.pdf

9. Bursey CR, Brooks DR. 2011. Nematode parasites of five species of turtles from the area de Conservación Guanacaste, Costa Rica, with description of a new species of Falcaustra. Comp Parasitol 78: 107-119. doi: 10.1654/ 4431.1

10. Bush AO, Lafferty K, Lotz J, Shostak AS. 1997. Parasitology meets ecology on its own terms: Margolis et al. revisited. J Parasitol 83: 575-583. doi: $10.2307 / 3284227$

11. Carrascal Velásquez, J, Negrete H, Rojano C, Álvarez G, ChacónPacheco J, Linares J. 2014. Caracterización hematológica de hicoteas (Trachemys callirostris Gray, 1856) en Córdoba, Colombia. Rev Med Vet 28: 4355. doi: $10.19052 / \mathrm{mv} .3180$

12. Castaño-Mora OV. 2002. Libro rojo de reptiles de Colombia. Serie Libros Rojos de Especies Amenazadas de Colombia. Bogotá: Universidad Nacional de Colombia. $37 \mathrm{p}$.

13. [CVS] 2009. Corporación Autónoma Regional de los Valles del Sinú y San Jorge. Informe de Gestión. Montería: CVS. $194 \mathrm{p}$.

14. De la Ossa JL. 2003. Manejo de fauna silvestre tropical. En: Programa de desarrollo sostenible de la Región de la Mojana. Bogotá: Departamento Nacional de Planeación. p 62-74.

15. De la Ossa J, Riaño R. 1999. Guía para el manejo, cría y conservación de la hicotea o jicotea Trachemys scripta callirostris (Gray). Bogotá: Convenio Andrés Bello. Serie Ciencia y Tecnología N. ${ }^{\circ} 74.40 \mathrm{p}$.

16. Flint M, Patterson-Kane J, Mills P, Limpus C. 2009. A veterinarian's guide for sea turtle post mortem examination and histological investigation. Queensland, Australia: University of Queensland. $56 \mathrm{p}$.
17. García VZ. 2013. Frecuencia de parásitos de reptiles en cautiverio en diferentes colecciones del estado de Morelos. Tesis de pregrado. Cuernavaca, Morelos: Univ. Autónoma del Estado de Hidalgo. 75 p.

18. Guglielmone AA, Nava S. 2006. Las garrapatas argentinas del género Amblyomma (Acari: Ixodidae): distribución y hospedadores. Rev Inv Agropec 35: 133-153.

19. Hidalgo-Vila J, Diaz-Paniagua C, Ribas A, Florencio $M$, PérezSantiagosa N, Casanova JC. 2009. Helminth communities of the exotic introduced turtle, Trachemys scripta elegans in southwestern Spain: transmission from native turtles. Res Vet Sci 86: 463-465. doi: 10.1016/j.rvsc.2008.08.003

20. Hidalgo-Vila J, Martinez-Silvestre A, Ribas A, Casanova JC, PerezSantigosa N, Diaz-Paniagua C. 2011. Pancreatitis associated with the helminth Serpinema microcephalus Nematoda Camallanidae in exotic red-eared slider turtles Trachemys scripta elegans. J Wild Dis 47: 201-205. doi: 10.7589/00903558-47.1.201

21. Jones A. 2005. Family Cladorchiidae Fischoeder, 1901.En: JonesA, Bray R, Gibson DI (eds). Keys to the Trematoda. Vol 2. London: CABI Publishing. p 257-317.

22. Julca R, Casas E, Chavera A, Sánchez L, Sánchez N, Batalla L. 2014. Descripción anatomopatológica de lesiones por helmintos gastrointestinales en tortugas motelo (Chelonoidis denticulata). Rev Inv Vet Perú 25: 37-50. doi: 10.15381/rivep.v25i1.8466

23. Lenis C, García-Prieto L. 2009. Polystomoides magdalenensis $\mathrm{n}$. sp. (Monogenoidea: Polystomatidae), a parasite of buccal cavity of Trachemys callirostris callirostris (Testudinata: Emydidae) from Colombia. J Parasitol 95: 850-854. doi: 10.1645/GE-1977.1

24. Lenis C, Vélez I. 2011. Digeneans in Trachemys callirrostris callirostris and Podocnemis lewyana (Testudinata) from the Magdalena River, Colombia: description of Pseudonematophila n. 
gen. and amendment of Nematophila Travassos, 1934 (Cladorchiidae: Schizamphistominae). Zootaxa 3095: 49-62. doi: 10.11646/zootaxa.3095.1.5

25. Martin DR. 1972. Distribution of helminth parasites in turtles native to southern Illinois. Trans Ill Acad Sci 65: 611-67.

26. Martínez-Silvestre A, Soler-Massana J. 2008. Enfermedades infecciosas y parasitarias en tortugas. Consulta Difus Vet 150: 43-45.

27. Martínez-Silvestre A, Guinea D, Soler J, Ferrer D. 2013. Presencia del parásito autóctono Serpinema microcephalus (Nematoda: Camallanidae) en las tortugas Trachemys scripta y Pseudemys concinna asilvestradas en Barcelona. Bol Asoc Herpetol Esp 24: 48-50.

28. McAllister CT, Goldberg SR, Holshuh HJ. 1993. Spiroxys contorta (Nematoda: Spirurida) in gastric granulomas of Apalone spinifera pallida (Reptilia: Testudines). J Wild Dis 29: 509-511. doi: 10.7589/0090-3558-29.3.509

29. Mendivelso D, Montenegro O. 2007. Diagnóstico del tráfico ilegal y del manejo post decomiso de fauna silvestre en nuevo corporaciones autónomas regionales de Colombia. Acta Biol Col 12S: 125-127.

30. Mestra A, Requena K. 2004. Aislamiento e identificación de parásitos gastrointestinales en hicoteas Trachemys scripta callirostris en el departamento de Córdoba. Tesis de Pregrado. Montería: Univ. de Córdoba. $60 \mathrm{p}$.

31. Ministerio de Ambiente y Desarrollo Sostenible. 2017. Resolución No 1912 de 15 de septiembre de 2017. República de Colombia. [Internet]. Disponible en: https://www.minambiente.gov.co/ images/normativa/app/resoluciones/75res\%201912\%20de\%202017.pdf

32. Moravec F, Vivas-Rodriguez C, Scholz T, Vargas-Vazquez J, Mendoza-Franco E, Schmitter-Soto JJ, GonzálezSolis D. 1995. Nematodes parasitic in fishes of cenotes ( $=$ sinkholes) of the Peninsula of Yucatan, Mexico: Part 2. Larvae. Folia Parasitol 42: 199-210.
33. Moravec F, Vargas-Vázquez, J. 1998. Some endohelminths from the freshwater turtle Trachemys scripta from Yucatan, México. J Nat His 32: 455-468. doi: 10.1080/00222939800770241

34. Oi M, Araki J, Matsumoto J, Nogami S. 2012. Helminth fauna of a turtle species introduced in Japan, the redeared slider turtle (Trachemys scripta elegans). Res Vet Sci 93: 826-830. doi: 10.1016/j.rvsc.2011.10.001

35. Palacios I, Bakker J, Guevara A. 1999. Tráfico y aprovechamiento de iguana e hicotea en la Zona Caribe de Colombia. Bogotá: Latin America Environment Society. $80 \mathrm{p}$.

36. Pasmans F, Blahak S, Martel A, Pantchev N. 2008. Introducing reptiles into a captive collection: the role of the veterinarian. Vet J 175: 53-68. doi: 10.1016/j.tvj1.2006.12.009

37. Platt T. 1977. Brief note. A survey of the helminth fauna of two turtle Species from northwestern Ohio. Ohio J Sci 77: 97-98.

38. Rataj VA, Lindtner-Knific LR, Vlahovic K, Mavri U, Dovc A. 2011. Parasites in pet reptiles. Acta Vet Scand 53: 33. doi: 10.1186/1751-0147-53-33

39. Salízar P, Sánchez L. 2004. Primer registro para Perú de Nematophila grandis (Diesing, 1839, Travassos, 1934; Trematoda Diplodiscidae) en Podocnemis unifilis (Froshel, 1848) testudines, Pelomedusidae. Rev Peru Biol 11: 3740. doi: 10.15381/rpb.v11i1.2430

40. Schmidt GD, Roberts LS. 2000. Trematoda: form, functions, and classifications of Digeneans. In: Schmidt GD, Roberts LS (eds). Foundations of parasitology. $6^{\text {th }}$ ed. Boston: McGrawHill. p 209-233.

41. TTWG (Turtle Taxonomy Working Group), Rhodin AGJ, Iverson JB, Bour R, Fritz U, Georges A, Shaffer HB, van Dijk PP. 2017. Turtles of the world. Annotated checklist and atlas of taxonomy, synonymy, distribution, and conservation status. $8^{\text {th }}$ ed. Lunenburg, MA, USA: Chelonian Research Foundation and Turtle Conservancy. $292 \mathrm{p}$. 
42. Vargas Ramírez M, del Valle C, Ceballos CP, Fritz U. 2017. Trachemys medemi n. sp. from northwestern Colombia turns the biogeography of South American slider turtles upside down. J Zool Syst Evol Research 55: 326-339. doi: 10.1111/jzs. 12179

43. Vélez A. 1999. Guía en parasitología veterinaria. $2^{\circ}$ ed. Medellín: Exitodinámica. $306 \mathrm{p}$.

44. Vicente J, Rodrigues HI, Gomes DC, Pinto RM. 1993. Nematoides do Brasil. Parte III: Nematoides de Répteis.
Rev Bras Zool 10: 19-168. doi: 10.1590/ S0101-81751993000100003

45. Yarto JE. 2011. Alojamiento y problemas relacionados en reptiles: quemaduras, problemas digestivos y respiratorios. IV Congreso Sociedad Latinoamericana de Medicina de Emergencias y Cuidados Intensivos -LAVECCS. Santiago de Chile, Chile.

46. Wilkinson R. 2004. Clinical pathology. In: McArthur S, Wilkinson R, Meyer J (eds). Medicine and surgery of tortoises and turtles. Ames, USA: Blackwell Publishing. p 141-186. 